\title{
Fast character modeling with sketch-based PDE surfaces
}

\author{
Lihua You ${ }^{1}$ - Xiaosong Yang ${ }^{1}$. Junjun Pan ${ }^{2} \cdot$ Tong-Yee Lee $^{3} \cdot$ Shaojun Bian $^{1}$ • \\ Kun Qian ${ }^{4} \cdot$ Zulfiqar Habib $^{5}$ - Allah Bux Sargano ${ }^{5}$ - Ismail Kazmi ${ }^{6}$ • Jian J. Zhang ${ }^{1}$
}

Received: 19 July 2019 / Revised: 6 April 2020 / Accepted: 8 May 2020 /

Published online: 6 June 2020

(C) The Author(s) 2020

\begin{abstract}
Virtual characters are 3D geometric models of characters. They have a lot of applications in multimedia. In this paper, we propose a new physics-based deformation method and efficient character modelling framework for creation of detailed 3D virtual character models. Our proposed physics-based deformation method uses PDE surfaces. Here PDE is the abbreviation of Partial Differential Equation, and PDE surfaces are defined as sculpting force-driven shape representations of interpolation surfaces. Interpolation surfaces are obtained by interpolating key cross-section profile curves and the sculpting force-driven shape representation uses an analytical solution to a vector-valued partial differential equation involving sculpting forces to quickly obtain deformed shapes. Our proposed character modelling framework consists of global modeling and local modeling. The global modeling is also called model building, which is a process of creating a whole character model quickly with sketch-guided and template-based modeling techniques. The local modeling produces local details efficiently to improve the realism of the created character model with four shape manipulation techniques. The sketch-guided global modeling generates a character model from three different levels of sketched profile curves called primary, secondary and key cross-section curves in three orthographic views. The template-based global modeling obtains a new character model by deforming a template model to match the three different levels of profile curves. Four shape manipulation techniques for local modeling are investigated and integrated into the new modelling framework. They include: partial differential equation-based shape manipulation, generalized elliptic curve-driven shape manipulation, sketch assisted shape manipulation, and template-based shape manipulation. These new local modeling techniques have both global and local shape control functions and are efficient in local shape manipulation. The final character models are represented with a collection of surfaces, which are modeled with two types of geometric entities: generalized elliptic curves (GECs) and partial
\end{abstract}

Electronic supplementary material The online version of this article (https://doi.org/10.1007/s11042-02009060-9) contains supplementary material, which is available to authorized users.

Lihua You

lyou@bournemouth.ac.uk

Extended author information available on the last page of the article 
differential equation-based surfaces. Our experiments indicate that the proposed modeling approach can build detailed and realistic character models easily and quickly.

Keywords Virtual characters · Model building · Sketch-guided global modeling · Template-based global modeling $\cdot$ Local modeling techniques $\cdot$ Generalized elliptic curves $\cdot$ PDE surfaces

\section{Introduction}

Modeling of virtual characters is essential in many multimedia applications. Various sketchbased, template-based, and physics-based methods have been developed to raise efficiency, increase details, and improve realism of virtual character modeling.

Sketch-based modeling creates 3D models from freehand drawings. It tackles the weaknesses of heavy manual operations and low modelling efficiency met by mainstream 3D modelling animation packages such as Maya and 3D Max. A main problem for sketchbased modeling is how to generate detailed and realistic 3D models. Sketching from a single view cannot provide the shape information in other views. Due to this reasons, most sketch-based modeling systems [18] sketch from many views with a workflow "sketchrotate-sketch" [12]. For such a workflow, how to find a good view for a stroke is often difficult and time-consuming, and due to the changes, users cannot match their input strokes to a guided image as pointed out in [12]. Creating an isometric view from a standard 3-view orthographic drawing avoids the difficulty of finding good views and has been popularly applied in engineering drawing and design [29]. It has also been used to create 3D man-made models [34]. However, one main limitation for this approach is with 3D organic models such as character models where a majority of facets share no common axes. Another limitation is it does not follow the "coarse-to-fine" workflow of organic modeling. The approach proposed in this paper is motivated by using three orthographic silhouettes of the whole model and its part models, but uses a stepwise approach to suit the "coarse-to-fine" workflow of organic modeling.

Although sketch-based approaches reduce manual operations and raise modeling efficiency, they are less capable in creating detailed and realistic 3D models. In order to address this issue, template-based approaches have been combined with sketch-based modelling to generate more detailed and realistic 3D models.

Most research activities investigate how to retrieve a 3D template model to match 2D sketches [10] and deform a 3D template model to fit the silhouette in one single view [21, 22]. In this paper, we introduce our proposed stepwise approach into template-based global modelling to deal with the silhouettes in three orthographic views which deforms a template $3 \mathrm{D}$ model to fit $2 \mathrm{D}$ primary profile curves, and uses secondary profile curves and key cross-section profile curves to further modify the deformed template model.

Physics-based deformations can greatly improve realism. Unfortunately, they usually involve heavy numerical calculations [31]. Such a weakness makes these physics-based deformations not ideal for real-time shape manipulations. Partial differential equation (PDE)based modeling is based on the equations similar to bending of elastic thin plates which involve a sculpting force to achieve physics-based deformations. Different from the physicsbased deformations which involve heavy numerical calculations, PDE-based modeling can greatly raise computational efficiency by using light analytical solutions to PDEs. However, obtaining analytical solutions of PDEs is a difficult task and current analytical solutions of 
PDEs are confined to the deformation regions between two separate boundaries, i. e., solving PDEs subjected to the constraints at two separate boundaries defined by two parametric values $u=0$ and $u=1$ (2-sided patches) [40] where $u$ is a parametric variable. In many applications, deformations are in the regions encircled by a closed boundary curve. It requires to solve PDEs subjected to the constraints at four boundaries defined by parametric values $u=0, u=1, v=0$, and $v=1$ (4-sided patches) where $v$ is a parametric variable. Since analytical solutions for PDE-based 4-sided patches are very difficult to obtain, analytical PDE-based deformations of 4-sided patches have not been solved in the existing work. In this paper, we will develop a new approach to tackle this unsolved problem, achieve analytical PDE solutions to carry out efficient physics-based deformations within the regions of both 2-sided and 4-sided patches, and investigate degenerated PDE surfaces called generalized elliptic curve-based interpolation surfaces which are generated from key cross-section profile curves.

With the proposed sketch-guided, template-based, and PDE-based techniques and generalized elliptic curve-based interpolation surfaces, we investigate four local modeling techniques to achieve shape manipulations efficiently. They are: partial differential equation-based shape manipulation, generalized elliptic curve driven shape manipulation, sketch-assisted shape manipulation, and template-based shape manipulation.

The contributions of this paper can be summarized as: (1) sketch-guided global modeling, (2) template-based global modeling, (3) analytical PDE-based deformations, (4) generalized elliptic curve-based interpolation surfaces, and (5) local shape manipulation techniques.

\section{Related work}

The work given in this paper is closely related to sketch-based modelling, template-based modeling, and physics and PDE based deformations. In what follows, we briefly review them.

\subsection{Sketch-based modeling}

Various sketch-based techniques have been developed. Comprehensive literature surveys of sketch-based modeling are made in [9, 33].

Most sketch-based modelling approaches such as [32, 46] use a "sketch-rotate-sketch" workflow. It allows users to draw sketches in multiple views to create $3 \mathrm{D}$ details and increase realism, and has been used in modeling of 3D organic models. As pointed out in [12], the weaknesses of these modeling approaches are: 1) users cannot trace a guide image, and 2) rotating and sketching from new views is the most difficult aspect of these approaches. Unlike organic models, most 3D man-made models can be directly defined from the silhouettes in three orthographic views: front, side and top [29, 34]. Another approach proposed in [39] is to generate a $3 \mathrm{D}$ curve network and create a $3 \mathrm{D}$ man-made model from the $3 \mathrm{D}$ curve network.

Recent work introduces machine learning into sketch-based modeling. Based on a convolutional network trained to map sketches to 3D shapes, Lun et al. investigate a 3D reconstruction method to create $3 \mathrm{D}$ shapes from $2 \mathrm{D}$ sketches [28]. The method is applicable to both a single sketch and multiple sketches. When only one single sketch is input each time, the convolutional network is trained to give a 3D shape. Then users add another sketch to refine the $3 \mathrm{D}$ shape. The process is repeated until a satisfactory $3 \mathrm{D}$ shape is obtained. When multiple sketches from different viewpoints are input, the convolutional network is trained to create a 3D shape from the input multiple sketches. By designing a convolutional neural network based 
deep regression network for inferring 3D face models from 2D sketches which fuses the convolutional neural network and shape based features of the input sketch, Han et al. propose a deep learning based sketching system for 3D face and caricature modelling which has a laborefficient sketching interface and supports gesture based interactions for users to further manipulate initial face models [16]. By training a convolution neural network on a large dataset generated by rendering sketches of various 3D shapes, incorporating the convolution neural network into the sketch processing workflow to infer the depth and normal maps representing a surface, introducing an intermediate convolution neural network layer to model the dense curvature direction or flow field of the surface, and producing an additional output confidence map along with depth and normal, $\mathrm{Li}$ et al. developed a method to create generic freeform 3D surfaces from sparse, expressive 2D sketches [24]. By introducing a novel sketch metaphor to create Poisson's equation-based freeform surfaces, implementing a convenient and flexible user interface, and using sparse strokes to achieve rapid modeling, Liu et al. presented a new approach to create 3D models with rich details [26]. In order to offer a rich source of information for a variety of computer-aided design tasks, Gryaditskaya et al. build a database of product design sketches called OpenSketch [13]. It contains more than 400 sketches representing 12 man-made objects drawn by 7 to 15 product designers of varying expertise and registers each sketch to its reference 3D model by annotating sparse correspondences. In order to learn priors from data, Smirnov et al. present a deep learning-based system for man-made shapes to infer a deformable parametric template model composed of Coons surface patches and introduce a synthetic sketch augmentation pipeline and a loss function for outputting non-self-intersecting shapes [35]. Through exploiting variational autoencoders to learn the mapping between a simple 2D stick figure sketch and the output 3D model and the embedding space around the model, Akman et al. propose the first method to create a 3D human models from a single sketched stick fig. [1].

Our proposed sketch-guided global modeling approach generates three different levels of 3D models directly from three different levels of profile curves. An initial model with circular cross-section curves is created from the primary profile curves. It is changed into an intermediate model by deforming the initial model to fit the secondary profile curves represented with elliptic cross-section curves. The final detailed model is obtained by fitting user-drawn key cross-section profile curves which deforms the intermediate model to change the elliptic crosssection curves into the user-drawn real key cross-section profile curves.

\subsection{Template-based modeling}

Template-based modeling includes generating a 3D template model and deforming the template model to match user-drawn sketches.

A main method of generating 3D template models is sketch-based shape retrieval. Various sketch-based shape retrieval methods have been reviewed in [23]. According to [23], existing sketch-based 3D model retrieval techniques can be categorized into: local 2D features versus global 2D features, bag-of-words framework versus direct shape feature matching, fixed views versus clustered views, and with view selection versus without view selection.

Most of recent methods involve machine learning. Through learning structural groups from a large database of scenes, $\mathrm{Xu}$ et al. developed a framework to automatically obtain semantically valid and well-arranged scenes of 3D models from a freehand sketch drawing which infers multiple scene objects [38]. Based on a novel data structure for fast retrieval of partial matches, Guo et al. investigated an on-demand part extraction algorithm called CustomCut 
[14]. For a user's input sketch query, it searches a database to find partially matching shapes, and identifies and extracts the region optimally matching the query in each shape to produce a customized part. Sketch-based shape retrieval for procedural modelling was also investigated in [17]. With the approach developed in the paper, user's hand-drawn 2D sketches are mapped to procedural model parameters through training a deep convolutional neural network by large amounts of automatically generated synthetic line drawings. These procedural model parameters are used to generate multiple, detailed output shapes that resemble the user's input sketches. In order to take advantage of both global and local features, Li et al. proposed a composite feature-based method to combine global features with local features which are extracted from representative 2D views of 3D models [25]. The global feature is used to select more relevant candidate models to the query sketch and the local feature is used to refine the selected candidate models. In [44], a framework is proposed to extract a sketch-based local binary pattern feature, determine the best view of a shape based on multilayer perceptrons (MLPs), project 3D models into many multiview images, use transfer learning to obtain the relationship between view images and sketches, and achieve shape retrieval based on two Siamese MLP networks. In [45], a deep learning technique is developed for sketch-based shape retrieval. The technique uses a sketch augmentation method to avoid simple image transformation and more closely mimic human sketches, introduces a convolutional neural network to learn the best viewing positions that best depict a 3D shape, and employs two Siamese convolutional neural networks to develop a cross-domain learning strategy that pairs up sketches and the 2D shape images. In [27], a framework is proposed to retrieve 3D models from a user provided free-hand sketch. In order to obtain plausible shapes contained in a sketch, a collection of shapes in the same category is used to train a shape space, and two different intuitive editing modes based on the shape space are implemented into a user interface for shape editing.

After using one of the above methods to obtain a suitable 3D template model, a new 3D model can be generated by deforming the template model to match user-drawn sketches. The main work on this topic uses user-drawn silhouette in a single view as a deformation constraint to deform the $3 \mathrm{D}$ template model such as in [22] which aligns user-drawn contour and a template 3D model, determines the optimal correspondences between the contour and the vertices on the template model, and uses the mean value coordinates to deform the $3 \mathrm{D}$ template model to match the user-drawn contour. In [21], a new approach is proposed which combines skeleton-based deformation with mesh editing to deform a 3D template model to fit user's drawn silhouette in a single view more efficiently.

The approach proposed in this paper differs from the work given in [21, 22]. Apart from deforming a template $3 \mathrm{D}$ model to match the primary profile curves in a single view, it adds the secondary profile curves and key cross-section profile curves to further deform the model to match all user-drawn profile curves in three orthographic views. Such an approach can create more detailed and realistic $3 \mathrm{D}$ models.

\subsection{Physics and PDE based deformations}

Physics-based deformations have been introduced into computer graphics about three decades ago. According to the comprehensive survey of various physically based deformable models used in computer graphics, most significant methods include: finite element/difference/volume methods, mass-spring systems, meshfree methods, coupled particle systems and reduced deformable models based on modal analysis [31]. 
Recently, some new methods were introduced into computer graphics. For example, a finite element method based on an elasto-plastic material model was implemented in [30], projective dynamics was developed in [8], a co-rotated linear finite element method was employed to handle large deformations of soft objects in [37], an example-based plasticity model based on linear blend skinning was proposed in [20], and a boundary element based method was investigated in [15] for fast simulation of brittle fracture. All these physics-based deformation methods involve heavy numerical calculations.

Partial differential equations (PDEs) have been widely used to describe various physical phenomena. Geometric modeling and shape deformations using PDEs can be regarded as physics-based and obtained by solving a vector-valued partial differential equation subjected to a set of additional constraints called the boundary conditions. Such a problem is a boundary value problem. Many methods have been developed to solve various boundary value problems. By using a continuous genetic algorithm, Arqub and Abo-Hammour present an efficient solver to solve systems of second-order boundary value problems [3]. The finite difference approximation is used to represent the derivatives of the differential equations in the systems, and the total residue of all nodes in the systems of second-order boundary value problems is formulated. The total residue is minimized, or the fitness function is maximized to obtain the solution to the systems. Based on the assumption of strongly generalized differentiability and the reproducing kernel theory, Arqub et al. investigate an iterative technique to obtain the analytic and approximate solutions of second-order, two-point fuzzy boundary value problems [4]. Through constructing the orthogonal basis from the obtained kernel functions where the constraint initial condition is satisfied, Arqub develop exact and numerical solutions to fuzzy Fredholm-Volterra integrodifferential equations with the reproducing kernel Hilbert space method [2]. By applying the theory of functional connections to embed the differential equation constraints into a constrained expression containing a free-function which is a linear combination of orthogonal basis functions with unknown coefficients and using linear/nonlinear least-squares to determine the unknown coefficients, Johnston et al. obtain the solutions to eighthorder boundary-value problems [19].

Different from existing physics-based deformation methods involving heavy numerical calculations, analytical solutions of PDE-based geometric modelling and shape deformations may be obtainable and have been used in creation of surface [43] and solid [42] models and surface blending [41]. They are much more efficient and simpler to implement than numerical PDE-based approaches $[6,7,36]$. These analytical solutions of PDEs are subjected to the constraints at two separate boundaries (2-sided patches) [40]. However, many shape deformations are within the regions encircled by a single closed boundary curve which can be divided into 4 boundaries (4-sided patches). How to develop analytical solutions of PDE-based shape deformations for 4-sided patches has not been solved. In this paper, we will tackle this problem.

In what follows, the mathematical model consisting of PDE surfaces and generalized elliptic curve-based interpolation surfaces is investigated in Section 3. Global modeling consisting of sketch-guided global modeling and template-based global modeling is proposed in Section 4 to generate a whole 3D character model quickly. Local modeling techniques are presented in Section 5 to create local 3D details, and experiments are made in Section 6. Finally, conclusions, limitations, and future work are discussed in Section 7. 


\section{Mathematical model}

The mathematical model discussed below involves the descriptions of PDE surfaces consisting of deformations caused by sculpting forces and interpolation surfaces which interpolate key cross-section profile curves.

\subsection{Deformation surfaces}

The deformation surfaces are defined by an analytical solution developed below. Inspired by the governing PDE for the elastic bending of thin plates, we use the fourth order partial differential equations to determine the deformation surfaces, which are given below

$$
\begin{aligned}
& {\left[a_{t 1} \frac{\partial^{4}}{\partial u^{4}}+a_{t 2} \frac{\partial^{4}}{\partial u^{2} \partial v^{2}}+a_{t 3} \frac{\partial^{4}}{\partial v^{4}}\right] t(u, v)=P_{t}(u, v)} \\
& (t=x, y, z)
\end{aligned}
$$

where $u$ and $v$ are the parametric variables of the surface, $x, y$, and $z$ are the position components of the surface, $a_{t 1}, a_{t 2}$ and $a_{t 3}$ are shape parameters, $t(u, v)$ is a position function whose components are $x(u, v), y(u, v)$ and $z(u, v)$, and $P_{t}(u, v)$ is a sculpting force function whose components are $P_{x}(u, v), P_{y}(u, v)$, and $P_{z}(u, v)$.

When exact closed form solutions to PDE (1) are sought, different combinations of the shape parameters $a_{t 1}, a_{t 2}$ and $a_{t 3}$ lead to different closed form solutions. When using PDE (1) to determine deformation surfaces, the values of the shape parameters $a_{t 1}, a_{t 2}$ and $a_{t 3}$ must be specified and the combination determined by the specified values of the shape parameters $a_{t 1}, a_{t 2}$ and $a_{t 3}$ is unique. Accordingly, the closed form solution is unique whose unknown constants are uniquely determined by the boundary conditions discussed below.

Compared to the control point and surface point based shape manipulations, physics-based approaches manipulate surfaces by applying virtual sculpting forces to deform the surfaces. Since sculpting forces can be applied at any positions and in different ways, surface manipulation using sculpting forces is physically meaningful and offers flexibility.

Our deformation technique considers three important aspects: fast generation of deformation surfaces based on an approximate analytical solution, flexibility in specifying deformation regions with any complicated shapes and with various sculpting forces, and an easy to use interface for applying sculpting forces.

Many parts of a virtual character, such as arms, legs and torso, are closed in one parametric direction $v$, and open in the other direction $u$. When considering surface deformations in the whole range of $v$ parametric direction, the boundaries in $v=0$ and $v=1$ are coincident and no boundary constraints are required. However, when discussing the surface deformations in a local range of $v$ parametric direction, the boundaries in $v=$ 0 and $v=1$ are two different ones and the boundary constraints must be satisfied. Due to this reason, the analytical resolution of PDE (1) requires surface deformations to be divided into two different types to respectively tackle 2 -sided patches with $u=0$ and $u=$ 1 boundaries and 4-sided patches with $u=0, u=1, v=0$, and $v=1$ boundaries. For the first type, it can change in a whole or local range in $u$ direction but only covers a whole range in $v$ direction. For the second type, it can change within a local range in $v$ direction and keeps the ranges in $u$ direction unchanged. 
Once a deformation region has been specified, it is re-parameterized in a local coordinate system. For simplifying mathematical symbols, we still use $u$ and $v$ to denote the parametric variables in a local coordinate system below.

We consider three types of sculpting forces: concentrated sculpting forces, line distribution sculpting forces, and area distribution sculpting forces.

The vector-valued concentrated sculpting force $\mathbf{P}(u, v)$ has three components $p_{x}, p_{y}$ and $p_{z}$, and acts at any position $\left(u_{p 0}, v_{p 0}\right)$ where we use the subscript $p$ to indicate sculpting forces.

Since a line distribution sculpting force acting in any direction in a global coordinate system can always be transformed into that acting in a parametric direction of a local coordinate system, we here consider the line distribution sculpting force acting within a range $\left\{u_{p 0} \leq u \leq\right.$ $\left.u_{p 1}, v=v_{p 0}\right\}$ in $u$ parametric direction of a local coordinate system and taking a sine form below

$$
P_{t}(u, v)=p_{t} \sin \pi\left(u-u_{p 0}\right) /\left(u_{p 1}-u_{p 0}\right)
$$

where $t=x, y, z$, and the sculpting force function $P_{t}(u, v)$ reaches the maximum value $p_{t}$ at the position $\left\{u=\left(u_{p 1}-u_{p 0}\right) / 2, v=v_{p 0}\right\}$.

Similarly, if an area distribution sculpting force acts within a region $\left\{u_{p 0} \leq u \leq u_{p 1}, v_{p 0} \leq v \leq v_{p 1}\right\}$ and takes a sine form in both parametric directions, it can be described with the following equation

$$
P_{t}(u, v)=p_{t} \sin \pi\left(u-u_{p 0}\right) \sin \pi\left(v-v_{p 0}\right) /\left[\left(u_{p 1}-u_{p 0}\right)\left(v_{p 1}-v_{p 0}\right)\right]
$$

where the sculpting force function $P_{t}(u, v)$ reaches the maximum value $p_{t}$ at the position $\left\{u=\left(u_{p 1}-\right.\right.$ $\left.\left.u_{p 0}\right) / 2, v=\left(v_{p 1}-v_{p 0}\right) / 2\right\}$.

For the first type of deformations, we transform the three types of sculpting forces into a unified mathematical representation below

$$
P_{t}(u, v)=\sum_{m=1}^{M} \sin m \pi u\left[p_{t m}+\sum_{n=1}^{N}\left(\bar{p}_{t m n} \cos 2 n \pi v+\overline{\bar{p}}_{t m n} \sin 2 n \pi v\right)\right]
$$

where $M$ is the total terms of the sine series of the parametric variable $u$, and $N$ is the total terms of the Fourier series of the parametric variable $v$.

The unknown constants $p_{t m}, \bar{p}_{t m n}$ and $\overline{\bar{p}}_{t m n}$ for concentrated sculpting forces, and line and area distribution sculpting forces are given in Appendix A.

For the first type of surface deformations, the deformation boundaries are at $u=0$ and $u=1$. The positional and tangential continuity conditions at these two boundaries require

$$
\begin{array}{lll}
u=0 & t=0 & \frac{\partial t}{\partial u}=0 \\
u=1 & t=0 & \frac{\partial t}{\partial u}=0
\end{array}
$$

where $(t=x, y, z)$.

The solution corresponding to sculpting force (4) and boundary conditions (5) represents a deformation surface which has the following form

$$
\begin{aligned}
t(u, v)= & G_{t 0}(v)+u G_{t 1}(v)+u^{2} G_{t 2}(v)+u^{3} G_{t 3}(v) \\
& +\sum_{m=1}^{M} \sin m \pi u\left[c_{t m}+\sum_{n=1}^{N}\left(\bar{c}_{t m n} \cos 2 n \pi v+\overline{\bar{c}}_{t m n} \sin 2 n \pi v\right)\right]
\end{aligned}
$$

where $G_{t i}(v)(t=x, y, z ; i=0,1,2,3)$ are unknown functions, and $c_{t m}, \bar{c}_{t m n}$ and $\overline{\bar{c}}_{t m n}$ are unknown constants. 
Inserting Eq. (6) into boundary conditions (5) and solving for the four unknown functions, we obtain

$$
\begin{aligned}
t(u, v)= & \sum_{m=1}^{M}\left\{\pi m\left\{-\left(u-3 u^{2}+2 u^{3}\right)+\left[1-(-1)^{m}\right] u^{2}(u-1)\right\}\right. \\
& +\sin m \pi u\}\left[c_{t m}+\sum_{n=1}^{N}\left(\bar{c}_{t m n} \cos 2 n \pi v+\overline{\bar{c}}_{t m n} \sin 2 n \pi v\right)\right]
\end{aligned}
$$

Substituting Eq. (7) into PDE (1) and minimizing the error of the PDE, the unknown constants $c_{t m}, \bar{c}_{t m n}$ and $\overline{\bar{c}}_{t m n}$ are found to be

$$
\begin{aligned}
c_{t m} & =p_{t m} /\left(\pi^{4} m^{4} a_{t 1}\right) \\
\bar{c}_{t m n} & =-\bar{p}_{t m n} / f_{t m n} \\
\overline{\bar{c}}_{t m n} & =-\overline{\bar{p}}_{t m n} / f_{t m n}
\end{aligned}
$$

where

$$
\begin{aligned}
f_{t m n} & =\pi^{4}\left[4 m^{2} n^{2}\left(a_{t 2}+\pi^{2} n^{2} a_{t 3} / 3\right)\right. \\
& \left.-\left(m^{4} a_{t 1}+4 m^{2} n^{2} a_{t 2}+16 n^{4} a_{t 3}\right)\right]
\end{aligned}
$$

In Fig. 1, we depict the shapes of a PDE surface from the first type of surface deformations caused by three different types of sculpting forces.

For the second type of deformations, since surfaces in the $v$ direction are non-closed, new boundary conditions given below should be added to Eq. (5) to maintain positional and tangential continuities at the four boundaries of the surfaces

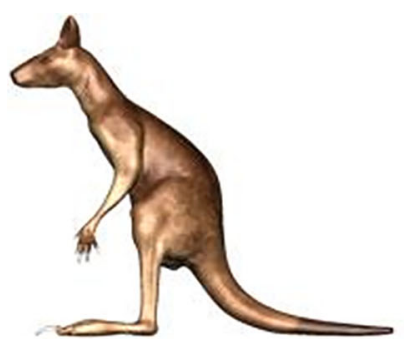

(a)

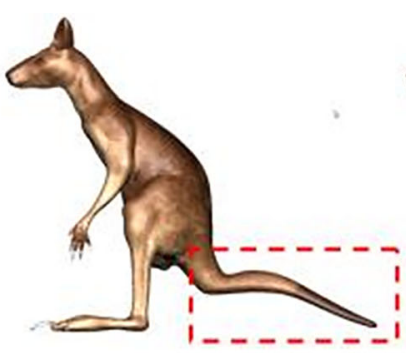

(b)

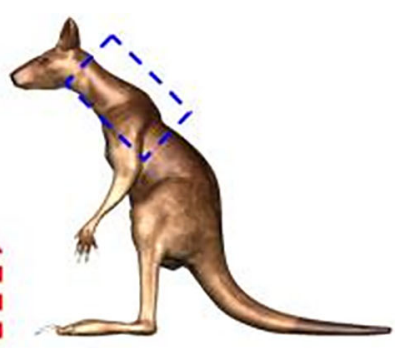

(c)

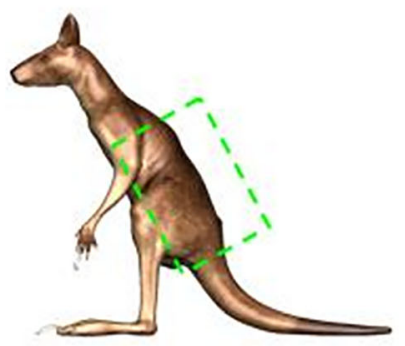

(d)

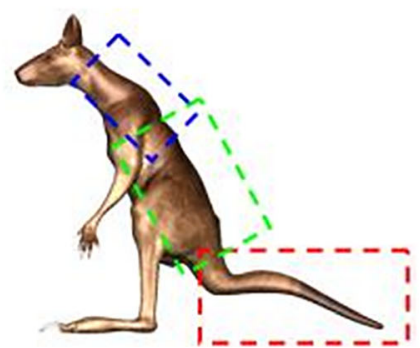

(e)

Fig. 1 First type of deformations. a Original model. b A concentrated sculpting force acts at the tail. c A line distribution force acts at the neck. $\mathbf{d}$ An area distribution force acts at the back. e Combination of deformations in (b), (c) and (d) 


$$
\begin{array}{lll}
v=0 & t=0 & \frac{\partial t}{\partial v}=0 \\
v=1 & t=0 & \frac{\partial t}{\partial v}=0
\end{array}
$$

where $t=x, y, z$.

In order to construct position functions which meet boundary conditions (5) and (10), we rewrite the three types of sculpting forces into the following form

$$
P_{t}(u, v)=\sum_{m=1}^{M} \sum_{n=1}^{N} p_{t m n} \sin m \pi u \sin n \pi v
$$

where $t=x, y, z, M$ is the total terms of the sine series of the parametric variable $u$, and $N$ is the total terms of the sine series of the parametric variable $v$.

The unknown constants $p_{t m n}$ for concentrated sculpting forces, and line and area distribution sculpting forces are given in Appendix B.

The solution which satisfies boundary conditions (5) and (10) exactly can be taken to be

$$
\begin{aligned}
t(u, v)= & v(1-v)\left[G_{t 0}(v)+u G_{t 1}(v)+u^{2} G_{t 2}(v)\right. \\
& \left.+u^{3} G_{t 3}(v)+\sum_{m=1}^{M} \sum_{n=1}^{N} d_{t m n} \sin m \pi u \sin n \pi v\right]
\end{aligned}
$$

where $G_{t 0}(v), G_{t 1}(v), G_{t 2}(v)$ and $G_{t 3}(v)$ are the unknown functions, and $d_{t m n}$ are unknown constants.

Substituting Eq. (12) into boundary conditions (5) and (10), we obtain

$$
\begin{gathered}
t(u, v)=v(1-v) \sum_{m=1}^{M} \sum_{n=1}^{N}\left\{\pi m \left\{-u+\left[2+(-1)^{m}\right] u^{2}\right.\right. \\
\left.\left.-\left[1+(-1)^{m}\right] u^{3}\right\}+\sin m \pi u\right\} d_{t m n} \sin n \pi v
\end{gathered}
$$

Introducing Eq. (13) into PDE (1) and minimizing the error of the PDE, we obtain $d_{t m n}$ below

$$
d_{t m n}=n^{2} p_{t m n} /\left(m \pi^{2} \bar{d}_{t m n}\right)
$$

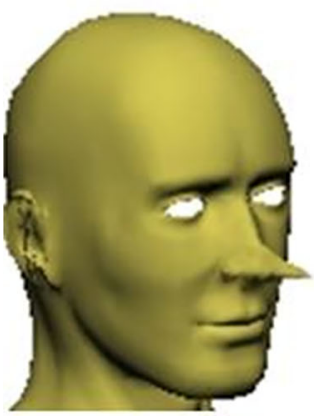

(a)

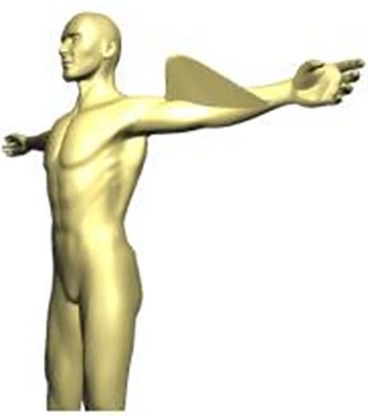

(b)

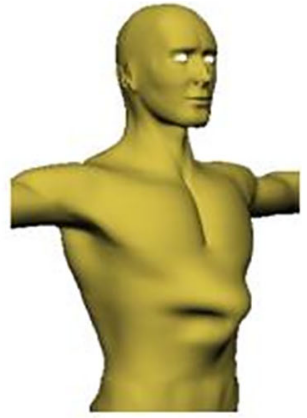

(c)

Fig. 2 Second type of deformations. a Nose deformation in a small region caused by a concentrated sculpting force. b Arm deformation in a very narrow curved band region caused by a line distribution sculpting force. c Chest deformation in a local region with an irregular boundary shape caused by an area distribution sculpting force 
where

$$
\bar{d}_{t m n}=2 m^{3} a_{t 1}+6 n^{4}\left(-m \pi^{2} / 12+1 / m\right) a_{t 3}
$$

In Fig. 2, we demonstrate shape changes in different deformation regions of a PDE surface from the second type of deformations caused by concentrated sculpting forces, and line and area distribution sculpting forces. With our proposed approach, these deformed shapes were created easily and quickly.

\subsection{Interpolation surfaces}

Interpolation surfaces can be regarded as a special case of PDE surfaces where the sculpting forces used to create deformation surfaces are zeroed.

Unlike the existing work of reconstructing surfaces from sampled planar cross-sections of arbitrary orientation [5] and non-parallel cross-section curves with genus-1 constraint [47], the interpolation surfaces introduced below are based on generalized elliptic curves and constructed from boundary curves and boundary tangents together with in-between key cross-section profile curves.

Key cross-section profile curves are the framework of an interpolation surface. In order to describe key cross-section profile curves with a unified mathematical representation, we introduce the conception of generalized elliptic curves which are defined by the following equation

$$
\begin{aligned}
& x=x_{0}+\sum_{j=1}^{J}\left(x_{2 j-1} \cos 2 j \pi v+x_{2 j} \sin 2 j \pi v\right) \\
& y=y_{0}+\sum_{j=1}^{J}\left(y_{2 j-1} \sin 2 j \pi v+y_{2 j} \cos 2 j \pi v\right) \\
& z=z_{c}
\end{aligned}
$$

where $J$ is the total terms of the cosine series and sine series, respectively, $z_{c}$ is the coordinate value of the key cross-section profile curve in zdirection, and $x_{k}$ and $y_{k}$ $(k=0,1,2,3, \cdots, 2 J)$ are unknown constants which can be determined by least squares curve fitting.

In Fig. 3, we make a comparison among the original key cross-section profile curves, corresponding generalized elliptic curves and standard ellipses where the curves in red, green and blue are the original ones, produced using our approach, and created with the standard ellipses, respectively. From the figure, big differences can be observed between the original
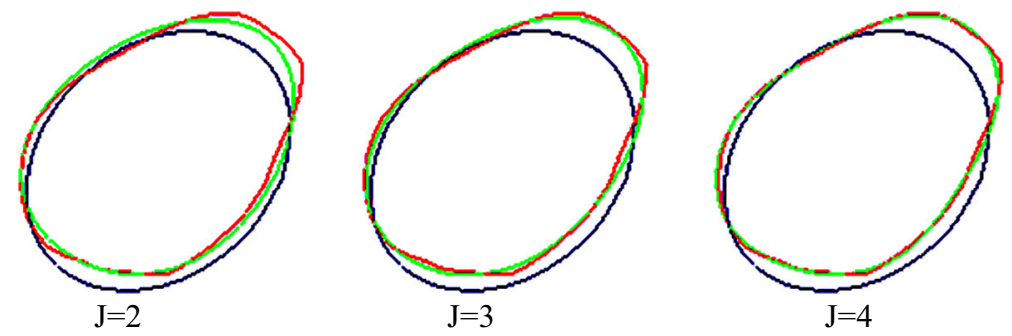

Fig. 3 Comparison among original key cross-section profile curves (red), generalized elliptic curves (green), and standard ellipses (blue) 


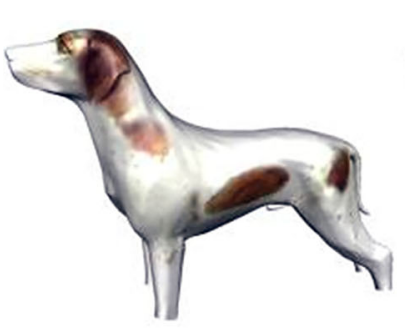

(a)

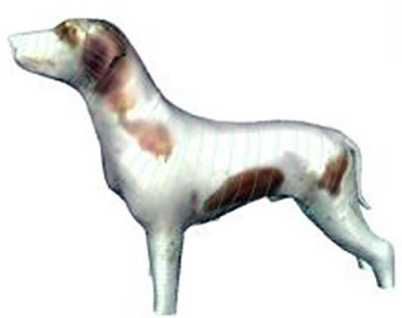

(b)

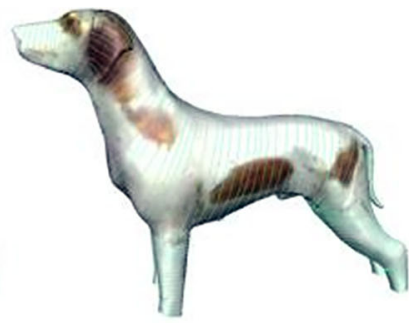

(c)

Fig. 4 Generalized elliptic curve-based interpolation surface. a Original model. b 150 GECs. c 240 GECs

ones and standard ellipses. In contrast, with small values of $J$, generalized elliptic curves approach the original curves very accurately. It indicates that generalized elliptic curves can represent key cross-section profile curves with high accuracy.

In Fig. 4, we give a dog model represented by interpolation surfaces with different numbers of generalized elliptic curves. It can be observed from the images in the figure that using a small number of generalized elliptic curves, we can obtain the interpolation surfaces which fit the original dog model very well. With the increase of generalized elliptic curves, the interpolation surfaces improve its modeling accuracy quickly.

\section{Global modeling}

Global modeling is the first stage of a modeling process. It is to create a whole model for further modifications to be made with local modeling.

For global modeling, we present two different modeling approaches: sketch-guided modeling and template-based modeling. With the former, the animator/modeler sketches the primary, secondary, and key cross-section profile curves of a 3D model, and the system creates the 3D model from these profile curves automatically. There is no need to refer to any existing models, and the animator has a total freedom to create the characters which they design. Therefore, it is especially suitable for the creation of grotesque characters such as monsters. With the latter, the primary, secondary, and key cross-section profile curves of a character are compared to those of a template model from a model database. This approach can create a detailed global model more efficiently and is more suitable for modeling of common characters. However, it requires a template model.

According to the above discussions, we can identify the following differences between sketch-guided global modeling and template-based global modeling.

1) Different global modeling process: Sketch-guided global modeling creates a new 3D character model directly from user's drawn sketches. However, template-based global modeling creates a new 3D character model by deforming a template character model to fit user's drawn sketches.

2) Different details: Sketch-guided global modeling can create rough models only since a single sketch in one view plane or several sketches in different view planes are not enough to define a 3D model with good details. In contrast, template-based global modeling makes use of good details of a template model and solves the problem that sketch-guided global modeling can create rough models only. 
3) Different flexibilities: Sketch-guided global modeling is more flexible in creating rough 3D shapes which users want. By comparison, template-based global modelling cannot create some special 3D models such as grotesque character models whose template models are not available.

\subsection{Sketch-guided global modeling}

Sketch-guided global modeling is undertaken through three different stages of modeling with an increasing level of details at each stage. As shown in Fig. 5 and the accompanied video, the first stage creates an initial global model from the primary profile curves; the second stage creates an intermediate global model with the secondary profile curves; and at the third stage, the final global model is refined with key cross-section profile curves.

Here the primary profile curves are the silhouette curves which are the most suitable for describing the whole shape of a 3D model. An intermediate global model is obtained by adding the constraints of secondary profile curves to the initial global model. The secondary profile curves are also some silhouette curves of a 3D model and its parts but viewed from the perpendicular directions to the first one. For 3D models with a complicated geometric shape, some of their parts can be removed to simplify the model, and the primary and secondary profile curves are produced from the simplified model. For the removed parts, their initial and intermediate global models are created from their own primary and secondary profile curves. A final global model is produced by modifying the key cross-section profile curves which are perpendicular to the primary and secondary profile curves and add the features which have not been characterized by the primary and secondary profile curves.

\subsubsection{Initial global models}

In order to create an initial global model, we sketch primary profile curves. For the modeling of a female character, we firstly sketch a primary profile curve as shown in Fig. 6a. Next, we identify the boundary curves which segment the female profile curve into different regions i.e., hair, arms, legs and torso, and determine the central curves between two profile curves of a region (Fig. 6b). The key cross-sections are then automatically located according to the variety of the curvature along the two profile curves, which can be manually edited if necessary. We

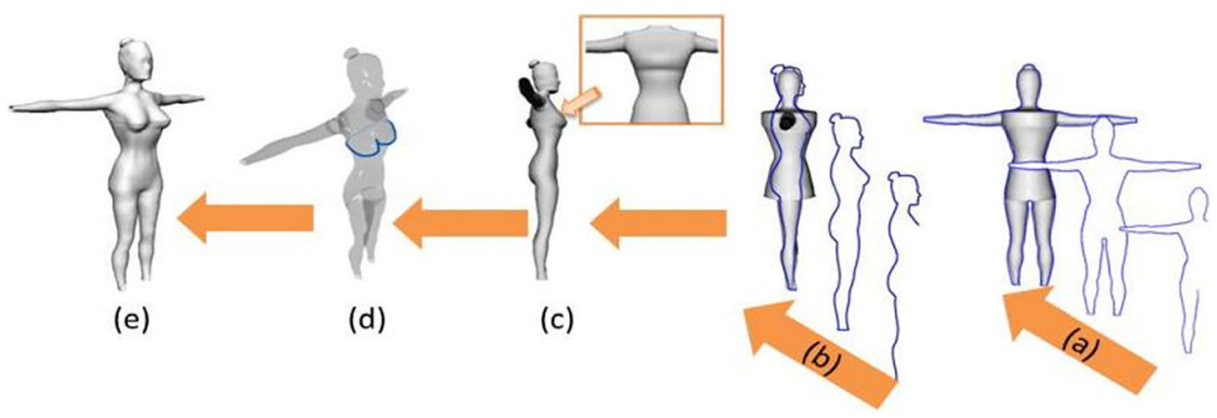

Fig. 5 Our proposed sketch-guided modeling quickly creates an initial global model from a profile curve in (a), uses a secondary profile curve in (b) to generate an improved intermediate global model in (c), and introduces key cross-section profile sketches in (d) to obtain a fine detailed global model in (e) 


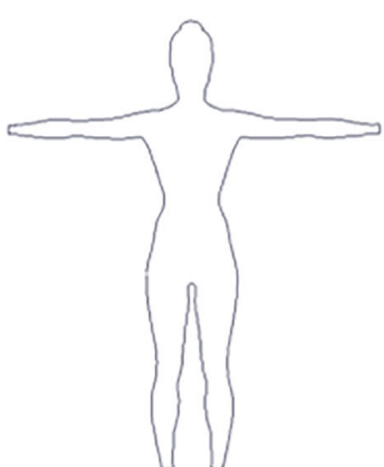

(a)

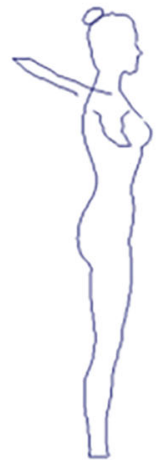

(d)

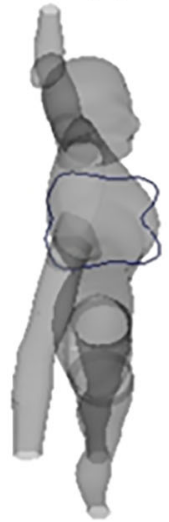

(h)

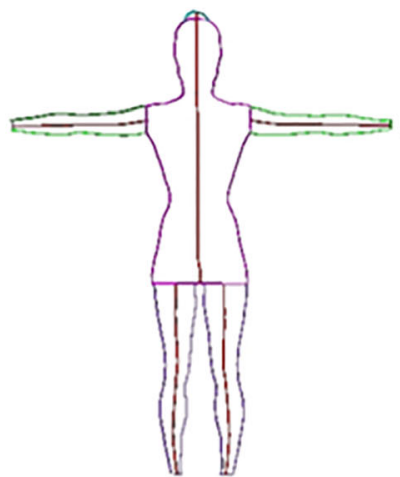

(b)

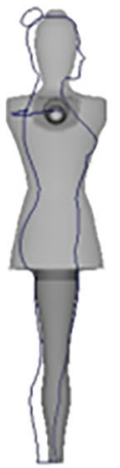

(e)

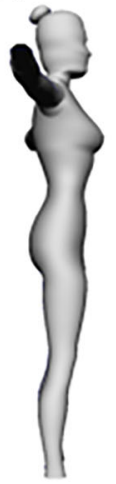

(f)

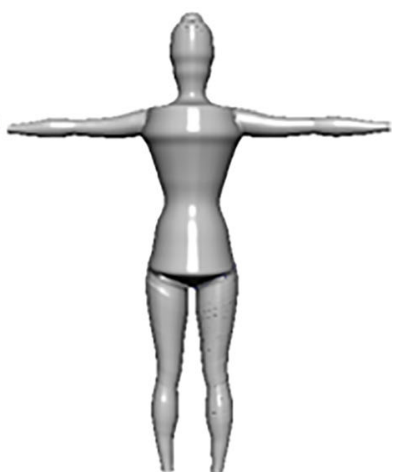

(c)

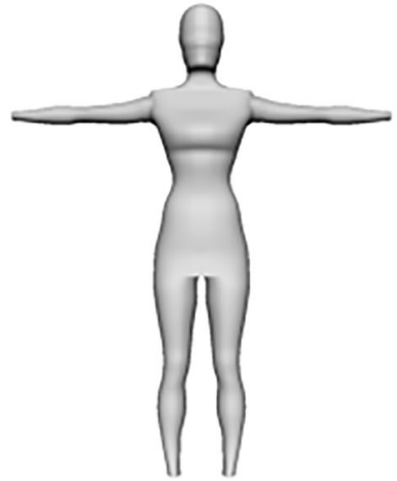

(g)

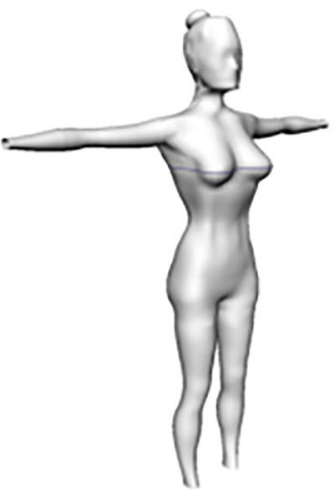

(i)

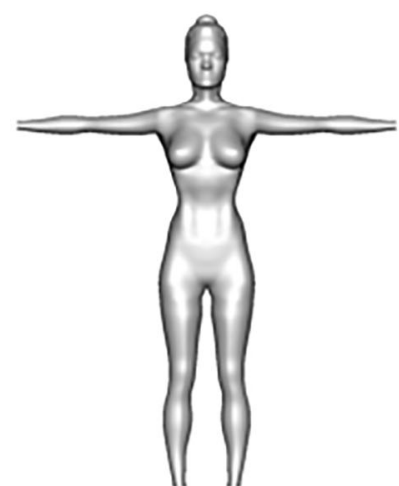

(j)

Fig. 6 Sketch-guided global modeling. a Primary profile curve. b Segmentation of primary profile curves and central curves. c Initial global model. d Secondary profile curves. (e) Secondary profile curves are applied on the initial global model. f, $\mathbf{g}$ Intermediate global model. $\mathbf{h}$ One key cross-section profile curve of the female is applied on the intermediate global model. i Breast of the female is created from the key cross-section profile curve. j Final global model is obtained by introducing more key cross-section profile curves

take circles to be the first approximation to the cross-section curves of the parts of the female model in these key cross-sections whose centers are situated on the central curves and 
diameters are determined by the distance between two profile curves of the parts. Then, the initial global model of each part is created and represented with a surface which interpolates these circles.

For the joints between legs, torso, and arms, the boundary curves between two adjacent parts are determined. Then, the tangents of surfaces at these two boundary curves are calculated. Finally, we create a smooth transition surface between these two boundary curves according to the information of the boundary curves and tangents at the boundary curves to smoothly connect the arm and leg surfaces to the torso surface. The image in Fig. 6c is a generated initial global model.

\subsubsection{Intermediate global models}

After an initial global model is obtained, the secondary profile curves shown in Fig. 6d are applied on the initial global model as indicated in Fig. 6e. Then, new positions of key crosssections, represented with ellipses, are obtained automatically according to the variety of the curvature along the primary and secondary profile curves. Once again, manual edit of the position of key cross-sections is allowed. Finally, the semi-major and semi-minor axes of the ellipses are calculated from the primary and secondary profile curves, and a new 3D model is created by interpolating these key cross-section ellipses.

The transition surfaces between different parts produced in the first stage are regenerated according to the boundary curves and tangents of the adjacent parts. The image in Fig. $6 \mathrm{f}$ and $\mathrm{g}$ indicates a generated intermediate global model.

\subsubsection{Final global models}

The cross-section contours obtained from the intermediate global models are standard ellipses. However, the real cross-section contours of 3D organic models are irregular curves which contain shape features of the models. In order to add these features to the intermediate global models, we sketch new contours at the positions of the key cross-sections or modify the existing contours of the character models in these key cross-sections. In this way, the ellipses at these key cross-sections are changed into the real cross-section profile curves. We use generalized elliptic curves to represent these real key cross-section profile curves. The final global models are produced and represented with PDE surfaces which interpolate these generalized elliptic curves and provide a powerful means for further local modeling. Figure $6 \mathrm{~h}$ demonstrates that although only one key cross-section profile curve of the female breast is applied on the intermediate global model, excellent approximation of the female breast is obtained as shown by Fig. $6 \mathrm{i}$.

By creating or modifying more key cross-section profiles, the final detailed global model is created and depicted in Fig. 6j.

A possible extension to the above treatment is that the secondary and key cross-section profile curves can be taken in arbitrary planes and directions.

\subsection{Template-based global modeling}

Template-based global modeling is to use the corresponding relationships between sketched profile curves and those of a template model with a similar topology to transform the template model into a new one represented with PDE surfaces which fits all the sketched profile curves. 
Fig. 7 Different character models built from template-based global modeling. An existing male model in (a) is changed into a fatter one in (b) and a boy in (c). An existing female model in (d) is changed into a pregnant woman in (e). An existing cow model in (f) is changed into a new model in (g). Among each group of the created $3 \mathrm{D}$ models, the left is from the primary profile curve, the middle is from the secondary profile curve, and the right is from the key cross section profile curves. The red primary profile of a male in (g) is used to change the cow model (f) into the right 3D model in (g)

Character models have complicated shapes, which cannot be completely and accurately defined with a $2 \mathrm{D}$ character sketch or some $2 \mathrm{D}$ character sketches in different view planes. A template character model has all the components and details of this type of character models. Templatebased global modeling creates a whole 3D character model by deforming a template character model to match user's drawn sketches. It has the property of making use of the components and details of the template character model, and the shape defined by the user's drawn sketches.

Similar to sketch-guided global modeling, Template-based global modeling can also be classified into three different global models: low-level global models, medium-level global models, and high-level global models which correspond to the model from the primary profile, primary and secondary profiles, and primary and secondary profiles plus key cross-section profile curves, respectively. The process of template-based global modeling is shown in the accompanied video and explained below.

\subsubsection{Low-level global modeling}

For low-level global modeling, we firstly sketch primary profile curves of a virtual character. With the method of shape matching and retrieval proposed in [11], we search a database to find a template model which has the primary profile curves fitting the sketched ones best.

Through geometrical transformations, the primary profile curves of the template model align with the sketched ones. Then, the radial basis function (RBF) interpolation is applied to deform the template model into a low-level model whose profile curves fit the sketched ones exactly.

The images in Fig. 7b and c demonstrate how a male in Fig. 7a is changed into a fatter one and a boy. Those in Fig. 7e demonstrate how a female in Fig. 7d becomes a pregnant woman. And the images in Fig. $7 \mathrm{~g}$ show the evolution of a cow in Fig. $7 \mathrm{f}$ to a different one. Among the created 3D models, those on the left are from primary profile curves.

\subsubsection{Medium-level global modeling}

For medium-level global modeling, secondary profile curves of the low-level model and its parts are first obtained. For the secondary profile curves to be modified, the corresponding new secondary profile curves are generated. By applying the geometric transformation operations and RBF interpolation which make the secondary profile curves of the low-level model and its parts fit the generated ones, the low-level model is changed into a medium-level model whose primary and secondary profile curves are the same as the generated ones. The medium-level 3D models from the medium-level global modeling are shown in the middle of Fig. 7b, c, e and g.

\subsubsection{High-level global modeling}

For high-level global modeling, we first decide the positions of the key cross-sections where the shape features of a virtual character at these key cross-sections have not been 

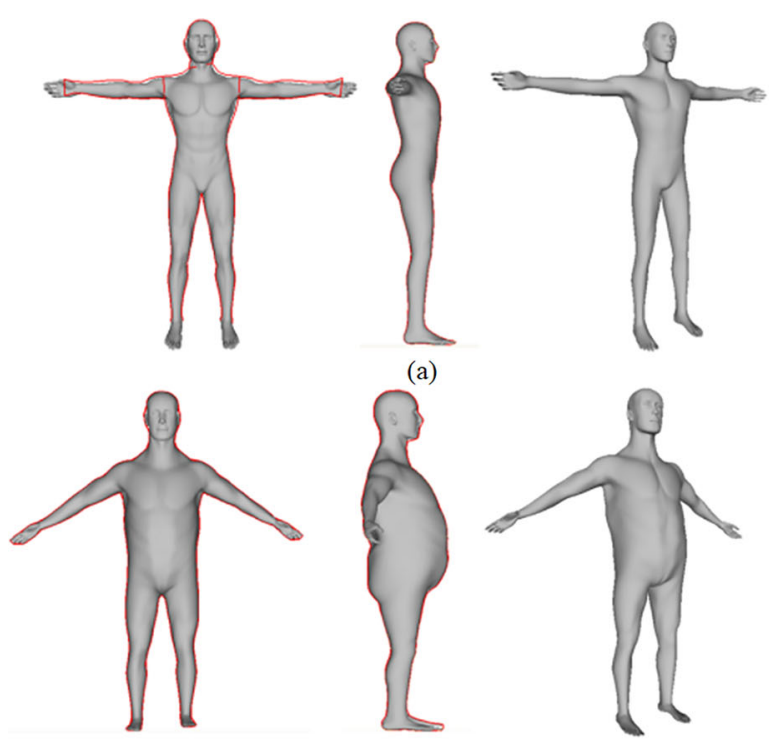

(b)
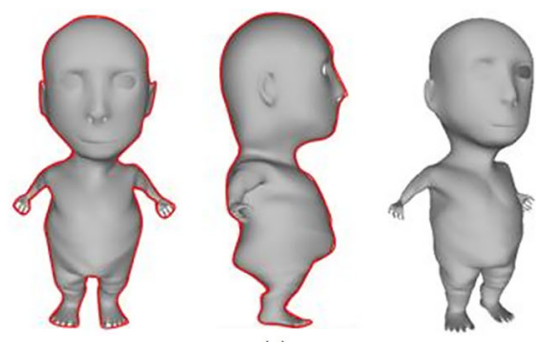

(c)
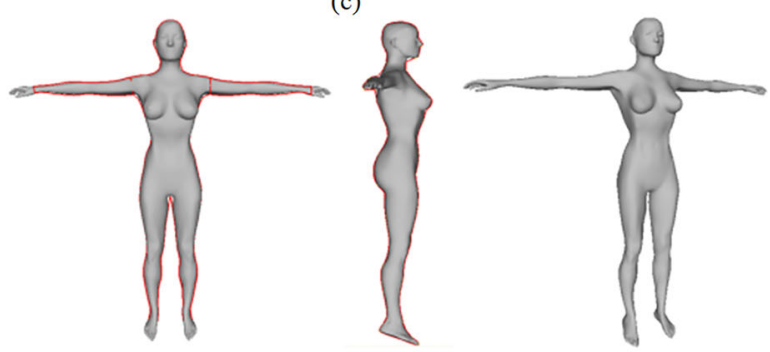

(d)
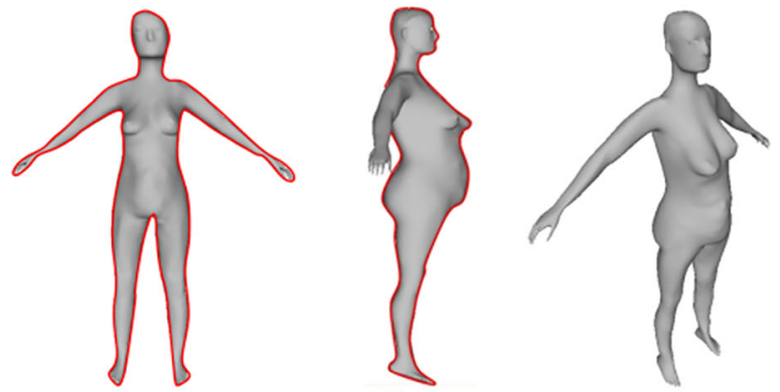

(e) 


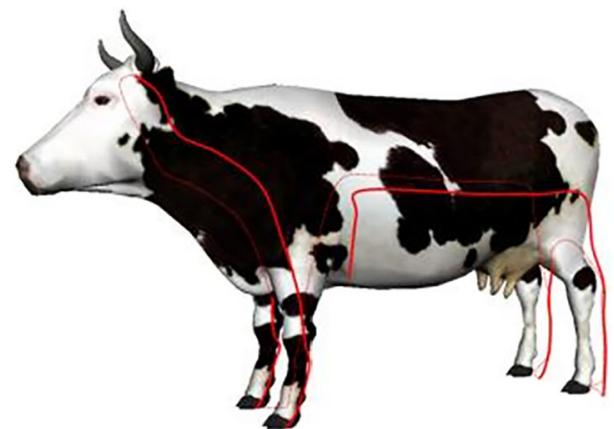

(f)
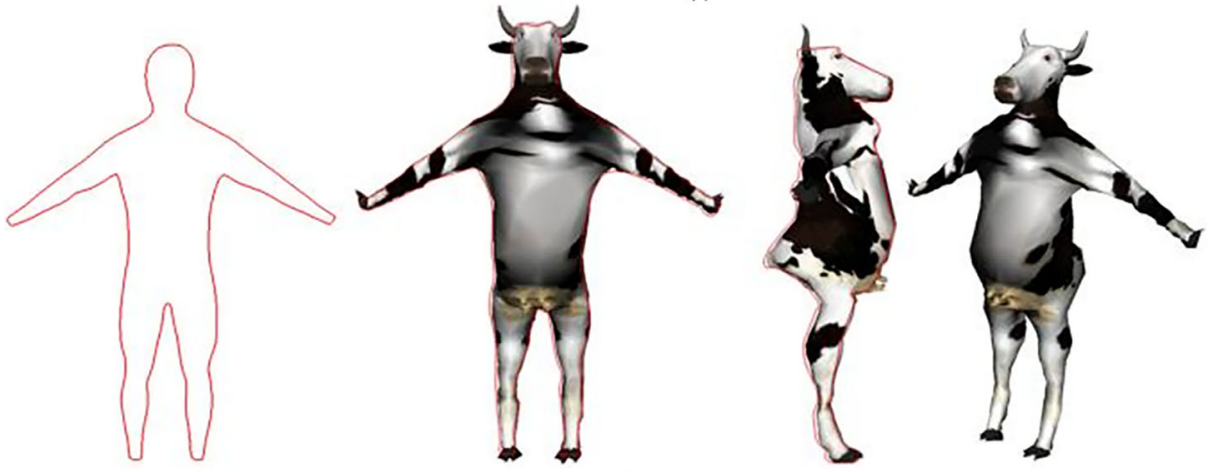

(g)

Fig. 7 (continued)

characterized by the medium-level model from the first two stages of the global modeling. Then, the key cross-section profile curves of the medium-level model are displayed and modified or new cross-section curves are created. Finally, RBF interpolation is employed to create new surface shapes which exactly fit all primary, secondary, and modified or new created key cross-section profile curves, and generate high-level global models. The created 3D models on the right of Fig. 7b, c, e and g are from high-level global modeling.

In the above, we use key cross section curves to create final global models and high-level global models. In fact, such a method can be extended to arbitrary spatial characteristic curves. That is to say, we can create final and high-level global models by making sketched spatial characteristic curves fit those of an existing model. For this extension, $z$ component in eq. (16) takes the form of trigonometric series to describe arbitrary spatial characteristic curves.

\section{Local modeling}

Local modeling is to create regional models or change local shapes to add $3 \mathrm{D}$ details. In addition to the existing shape manipulation approaches, we investigate four shape manipulation techniques. They include: shape manipulation by PDE surfaces, generalized elliptic curves, sketch-guided deformations, and template-based shape changes. 


\subsection{PDE based shape manipulation (PDESM)}

With our PDE based technique, three methods can change the shape of a surface. They are sculpting forces, shape parameters, and boundary curves and boundary tangents. Among these methods, sculpting forces are the most powerful and flexible in shape manipulation as introduced in Subsection 3.1 and shown by Figs. 1 and 2. Compared to the control point or surface point based shape manipulations, the sculpting force based shape manipulation has the following advantages:

(1) A sculpting force can be specified at any positions conveniently. In contrast, the shape manipulation operations of the control point or surface point based approach cannot be performed at the positions where no surface or control points exist.

(2) Deformation regions with any complicated boundary shapes can be easily specified with the sculpting force based shape manipulation. Oppositely, the control point and surface point based approaches are difficult to control a shape change which occurs within a prespecified region with a complex boundary. Such a function is very useful for some surface deformations. For example, if we want to model a human nose, the deformation should occur only within the boundary curve between the nose and the face which has an irregular geometric shape.

(3) Sculpting force based approach is very powerful in both global and local shape manipulations.

Apart from sculpting forces, shape parameters, boundary curves, and boundary tangents are also effective in shape manipulations as demonstrated below.

When non-zero boundary curves and tangents are to be considered, boundary conditions and the solutions of PDE (1) should be modified accordingly. Due to the limitation of space, we will not discuss this here.

By changing shape parameters, we can obtain different deformation shapes as depicted by Fig. $8 \mathrm{~b}$ and c. Figure $8 \mathrm{~d}$ and e give an example how boundary curves and boundary tangents affect surface shapes.

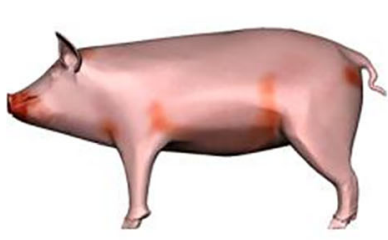

(a)

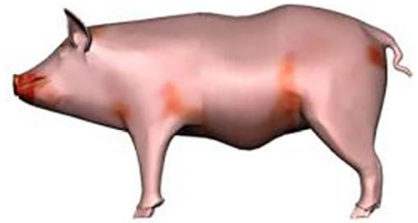

(b)

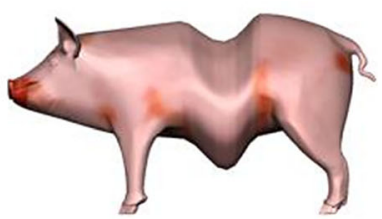

(c)

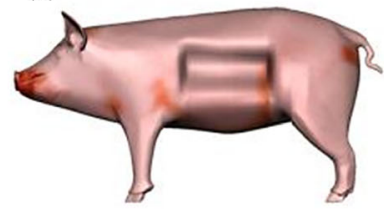

(d)

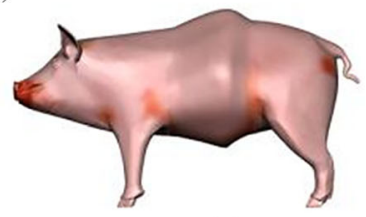

(e)

Fig. 8 PDE based surface manipulation of a pig torso. a Original model. b Deformation with shape parameters 1.0. $\mathbf{c}$ Deformation with shape parameters 0.3 . $\mathbf{d}$ Shape variation by changing boundary curves. e Shape variation by changing boundary tangents 


\subsection{Generalized elliptic curves driven shape manipulation (GECDSM)}

We can change the shape or size of generalized elliptic curves describing key cross-section profile curves, or perform geometrical transformations to achieve new shapes.

As shown in Fig. 9, the geometric transformations are applied to the generalized elliptic curves of the elephant trunk in Fig. 9a to change it into a new shape in Fig. 9b. The size of the generalized elliptic curves of the elephant torso is changed to obtain the torso shape shown in Fig. 9c. The local shapes of generalized elliptic curves of the elephant legs are changed to generate new leg shapes depicted in Fig. 9d.

\subsection{Sketch assisted shape manipulation (SASM)}

For sketch assisted shape manipulation, we present two methods: sketch on a 3D model, and auxiliary lines assisted sketches.

Sketch on a 3D model is to sketch a few local profile curves and generate a local model from these profile curves. For example, in order to create a female breast, we sketch a closed red curve on the chest of a female model and two blue profile curves of a woman breast on the same model as shown in Fig. 10a.

Auxiliary lines assisted sketches are to add some supplemental sketches and provide additional geometric information to define a local shape. For example, the profile curve of a human gives the main contour of a nose. By increasing an auxiliary sketch curve in bright green along the interface between the nose and face shown on the left of Fig. 10b, we create a rough shape of a human nose shown on the right.

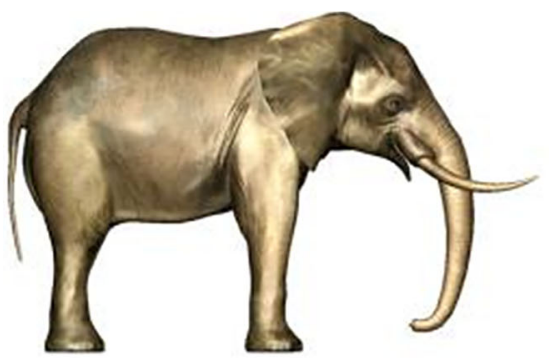

(a)

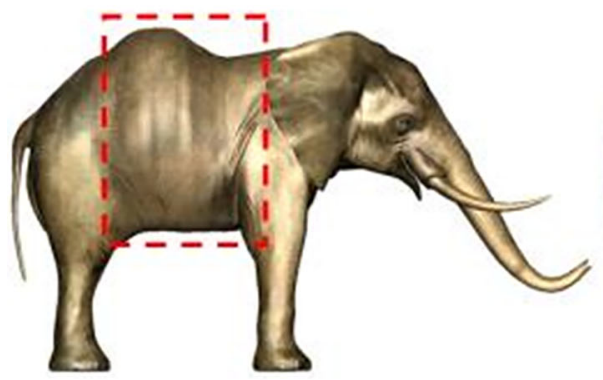

(c)

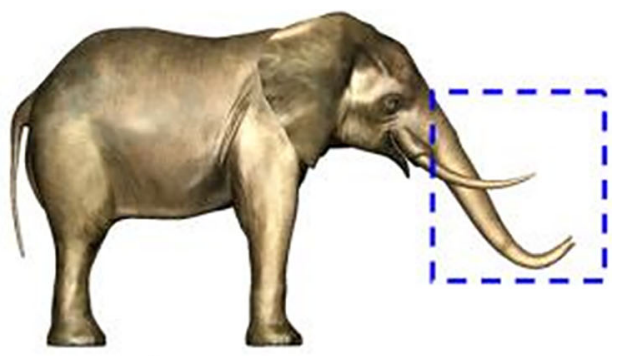

(b)

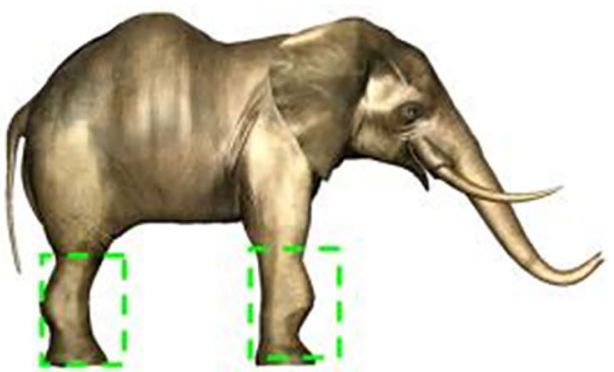

(d)

Fig. 9 Generalized elliptic curves driven shape manipulation. a Original model. b Deformation of the trunk by geometrical transformation. c Global deformation of the torso. d Local deformation of the legs 


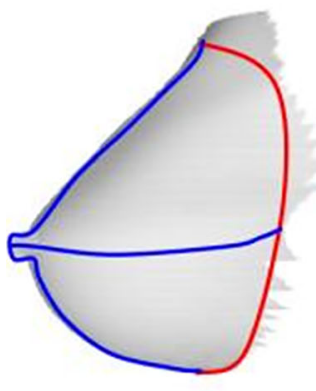

(a)
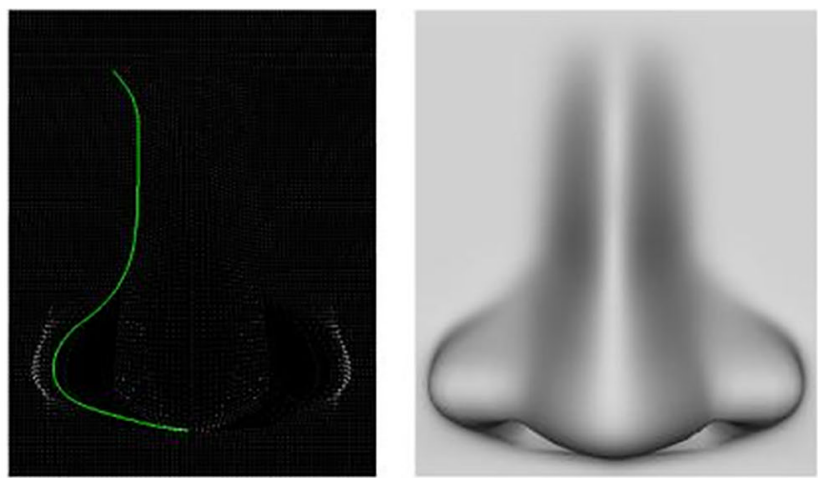

(b)

Fig. 10 Sketch assisted shape manipulation. a A female breast from sketches on a 3D model. b A human nose created by adding an auxiliary sketch curve

\subsection{Template-based shape manipulation (TBSM)}

Template-based shape manipulation includes local part creation through use of the corresponding relations between the created local profile curves and those of existing character parts and regional shape changes by comparing the created key cross-section curves to the corresponding ones of a template character model.

In Fig. 11a, we draw the profile curve of an ear on the right, use the corresponding relation between the profile curve and that of a similar model in our database shown on the left, and change the existing ear on the left into a new one on the right. In Fig. 11b, we compare the cross section curves in the middle, which are ellipses created by the sketch-based global modeling, to those of a template foot model on the left and change the foot model originally represented with ellipses in the middle into a new model on the right which has real cross section curves of a human foot.

Our proposed modeling system also represents a virtual character with both a PDE model and a polygon or NURBS model. With such a treatment, we can carry out shape manipulations on the PDE model with our proposed local modeling techniques, and do the work on the polygon or NURBS model using the existing local shape manipulation methods. Figure 12a and $\mathrm{b}$ indicate interrelated PDE and polygon representations of the same model.

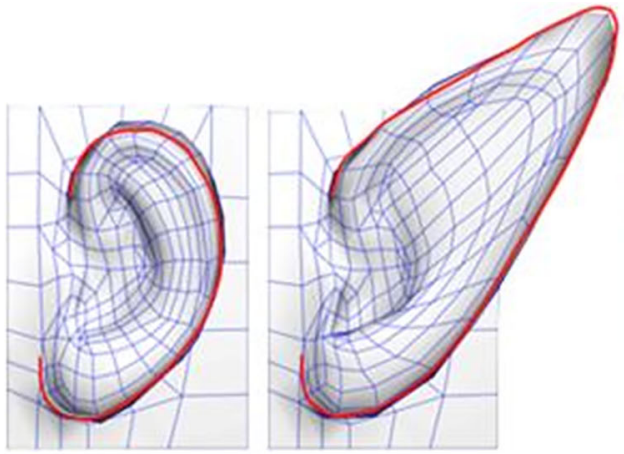

(a)
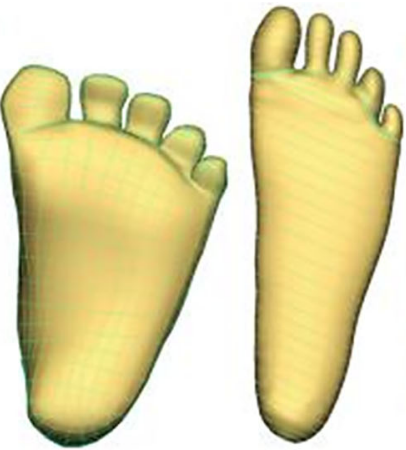

(b)

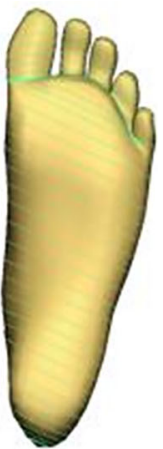

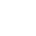

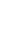

Fig. 11 Sketch-based shape manipulation. a Referring to an existing local part. b Referring to existing cross section curves 


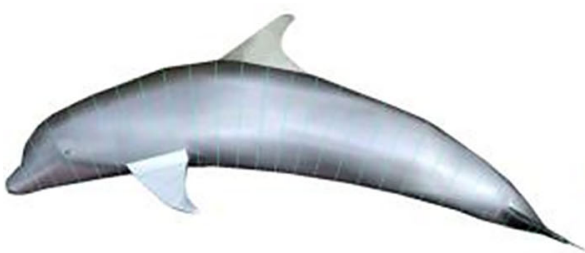

(a)

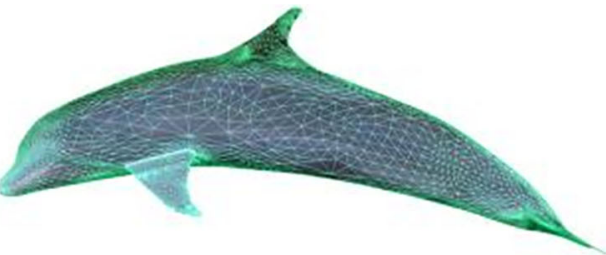

(b)

Fig. 12 PDE and polygon representations of a same model. a Generalized elliptic curves and PDE surfaces. b Polygon model with mesh

\section{Experiments}

Except for sketch-guided global modeling and template-based global modeling which were implemented differently, all the mathematical equations developed in Section 3 and local modeling techniques were implemented into two different versions. The first version uses Microsoft Visual C++ and OpenGL. The Autodesk Maya provides a powerful platform in drawing 3D sketches and quickly integrates PDE surface-based 3D models with polygon and NURBS modeling. The second version uses the Maya Python scripts. Template-based global modeling was implemented with Microsoft Visual C++ and OpenGL, and sketch-guided global modeling was implemented using Maya Python scripts.

With the implemented software, we carried out experimental work to compare our proposed approach to the existing ones.

Our first experiment is to examine the amount of information used to represent a same character model. For a polygon model given in Fig. 13a, the total polygon faces are 9451 and vertices are 6413. Using our proposed sketch-based PDE approach, we rebuilt the model shown in Fig. 13c which consists of 32 PDE surfaces determined by a total of 139 generalized elliptic curves shown in Fig. $13 \mathrm{~b}$ and 2224 coefficients. This experiment indicates our proposed approach requires much smaller data to represent a model, accordingly, greatly reduces the data storage and the demand for computer resources.

Our second experiment is to compare manual operations of shape manipulation with the sculpting force-based method proposed in Subsection 3.1 and by the vertex-based

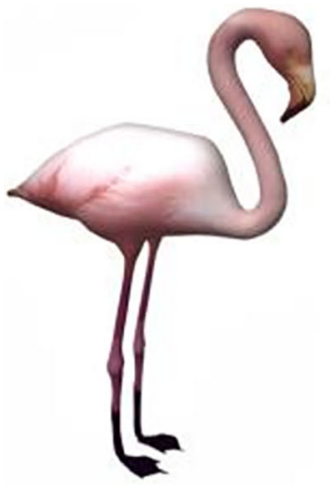

(a)

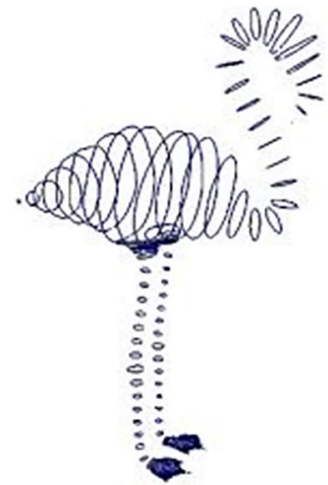

(b)

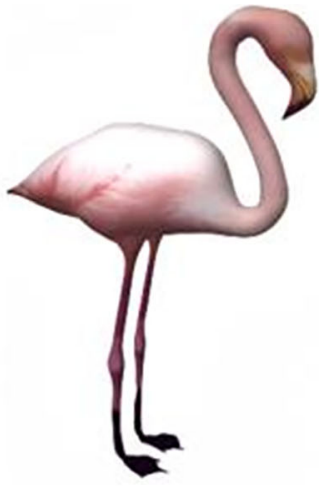

(c)

Fig. 13 Comparison of information amount used to represent a model. a Polygon model. b Generalized elliptic curves. c PDE model 


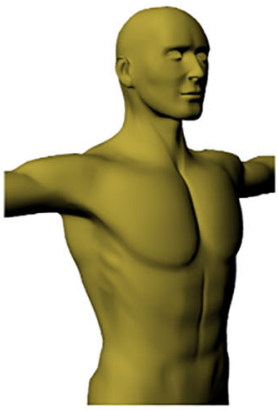

(a)

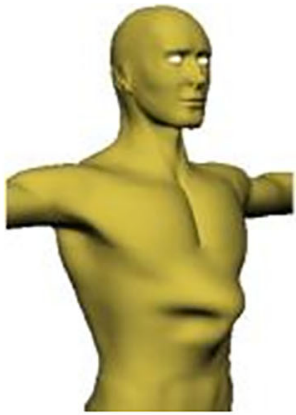

(b)

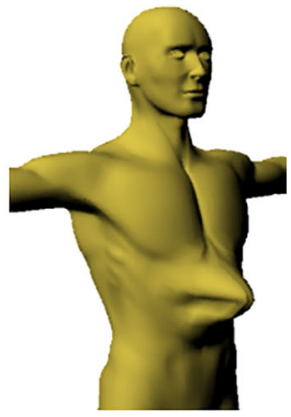

(c)

Fig. 14 Comparison of shape manipulation between sculpting force-based method of PDE deformation surfaces and vertex-based method of polygon models. (a) Undeformed shape. (b) Deformed shape achieved by sculpting force-based shape manipulation. (c) Deformed shape achieved by vertex-based shape manipulation

method which tweaks the vertices of polygon models. With the deformation surfaces proposed in Subsection 3.1, one manual operation was required to draw a closed curve on the chest surface of the 3D model shown in Fig. 14(a), the implemented sculpting force-based algorithm automatically parametrized the region enclosed by the drawn closed curve into $\{0 \leq u \leq 1,0 \leq v \leq 1\}$. After that, another manual operation was required to move a mouse and the movement was automatically changed into a sculpting force to create the chest deformation shown in Fig. 14(b). Since the chest deformation region has an irregular boundary, when tweaking the vertices of the 3D polygon model in the same deformation region, 15 manual operations were required to create a similar chest deformation in a similar deformation region shown in Fig. 14(c). This example indicates that the sculpting force-based shape manipulation proposed in this paper can greatly reduce manual operations and raise modeling efficiency.

Our third experiment is to investigate the efficiency of building a character model using different approaches. We ask an animator to build a same character model. He took about $10 \mathrm{~h}$ to build the model in Fig. 15a using polygons, and about $7 \mathrm{~h}$ to create the same model in

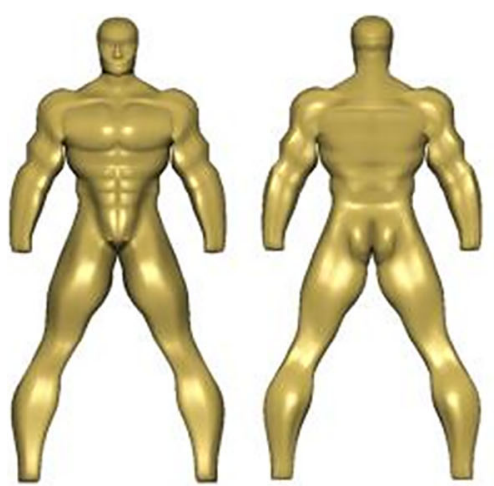

(a)
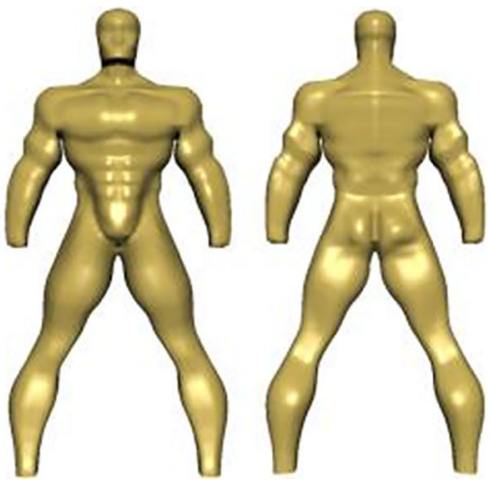

(b)

Fig. 15 Comparison of efficiency of different modeling approaches. (a) Polygon modeling. (b) Sketch-based PDE modeling 
Fig. 15b with our sketch-based PDE modeling. In the figure, those on the left are from the front view, and those on the right are from the back view.

\section{Conclusions, limitations, and future work}

In this paper, we have presented a new physics-based deformation method and modelling framework to create a global model of virtual characters from sketched primary profile curves and further modify it by using other profile curves and local shape modeling techniques. We have developed an analytical solution of PDE-based deformations for 4-sided patches, generalized elliptic curve-based interpolation surfaces, two global modeling approaches and four shape manipulation methods. The character modelling examples presented in this paper indicate that our proposed modelling framework can create various character models with good details and realism easily and quickly.

There are several extensions can be made from the approach proposed in this paper. First, all the examples used to demonstrate the proposed approach are some of organic models. No man-made models are used to demonstrate the proposed approach. It is a limitation of the proposed approach. Since man-made models have simpler shapes than organic models, the proposed approach should be applicable to man-made models as well. In the future, we intend to extend the proposed approach to modelling of other organic models and modify it to suit modeling of man-made models. Second, machine learning has been introduced into sketchbased modelling and template-based modelling. It can be also introduced into the sketchguided global modelling, template-based global modelling, and some of the local modelling techniques to further enhance the power of the techniques proposed in this paper. Third, the computer graphics packages such as the Autodesk Maya have a powerful plug-in feature to add new functions and increase their capacity. The approach proposed in this paper can create detailed 3D models easily and quickly. In order to maximize its use, we intend to implement all the algorithms of the proposed approach into a Maya plug-in so that more users would be able to use it. Fourthly, virtual reality is becoming more and more popular and how to create detailed 3D models easily and quickly in a virtual environment is an active topic. The proposed approach can be extended to address this issue. We intend to investigate this extension in the future.

Acknowledgements This research was supported by the PDE-GIR project which had received funding from the European Unions Horizon 2020 research and innovation programme under the Marie Skodowska-Curie grant agreement No 778035 and Innovate UK (Knowledge Transfer Partnerships Ref: KTP010860), and supported in part by the National Science Council of Taiwan under Grant No. 107-2221-E-006-196-MY3.

\section{Appendix 1: Determination of the unknown constants $p_{t m v} \bar{p}_{t m n}$ and $\overline{\bar{p}}_{t m n}$}

For area distribution sculpting forces,

$$
\begin{aligned}
p_{t m} & =-c_{0} p_{t} f_{1}(u, m) \\
\bar{p}_{t m n} & =-c_{0} p_{t} f_{3}(u, m) f_{2}(v, 2 n) \\
\overline{\bar{p}}_{t m n} & =c_{0} p_{t} f_{3}(u, m) f_{3}(v, 2 n)
\end{aligned}
$$


where

$$
\begin{aligned}
& f_{1}(u, m)=u_{0} \cos ^{2} m \pi u_{1}-u_{2} \cos ^{2} m \pi u_{3} \\
& f_{2}(u, m)=v_{0} \sin ^{2} 2 n \pi v_{1}-v_{2} \sin ^{2} 2 n \pi v_{3} \\
& f_{3}(r, t)=0.5\left(r_{0} \sin 2 k \pi r_{1}-r_{2} \sin 2 k \pi r_{3}\right) \\
& c_{0}=4\left(u_{p 1}-u_{p 0}\right)\left(v_{p 1}-v_{p 0}\right) / \pi^{2} \\
& r_{0}=1 /\left[k\left(r_{p 1}-r_{p 0}\right)+1\right] \\
& r_{1}=0.5\left(r_{p 1}+r_{p 0}+1 / k\right) \\
& r_{2}=1 /\left[k\left(r_{p 1}-r_{p 0}\right)-1\right] \\
& r_{3}=0.5\left(r_{p 1}+r_{p 0}-1 / k\right) \\
& (r=u, v ; k=m \text { for } r=u ; k=2 n \text { for } r=v)
\end{aligned}
$$

For line distribution sculpting forces,

$$
\begin{aligned}
& p_{t m}=c_{1} p_{t} f_{3}(u, m) \\
& \bar{p}_{t m n}=2 c_{1} p_{t} f_{3}(u, m) \cos 2 n \pi v_{p 0} \\
& \bar{p}_{t m n}=2 c_{1} p_{t} f_{3}(u, m) \sin 2 n \pi v_{p 0}
\end{aligned}
$$

where

$$
c_{1}=-2\left(u_{p 1}-u_{p 0}\right) / \pi
$$

For concentrated sculpting forces,

$$
\begin{aligned}
& p_{t m}=2 p_{t} \sin m \pi u_{p 0} \\
& \overline{\bar{p}}_{t m n}=4 p_{t} \sin m \pi u_{p 0} \cos 2 n \pi v_{p 0} \\
& \overline{\bar{p}}_{t m n}=4 p_{t} \sin m \pi u_{p 0} \sin 2 n \pi v_{p 0}
\end{aligned}
$$

\section{Appendix B: Determination of the unknown constants $p_{t m n}$}

For area distribution sculpting forces,

$$
p_{\text {tmn }}=c_{0} p_{t} f_{3}(u, m) f_{3}(v, k / 2)
$$

For line distribution sculpting forces,

$$
p_{\text {tmn }}=2 c_{1} p_{t} f_{3}(u, m) \sin n \pi v_{p 0}
$$

For concentrated sculpting forces,

$$
p_{\text {tmn }}=4 p_{t} \sin m \pi u_{p 0} \sin n \pi v_{p 0}
$$

Open Access This article is licensed under a Creative Commons Attribution 4.0 International License, which permits use, sharing, adaptation, distribution and reproduction in any medium or format, as long as you give appropriate credit to the original author(s) and the source, provide a link to the Creative Commons licence, and indicate if changes were made. The images or other third party material in this article are included in the article's Creative Commons licence, unless indicated otherwise in a credit line to the material. If material is not included in the article's Creative Commons licence and your intended use is not permitted by statutory regulation or exceeds the permitted use, you will need to obtain permission directly from the copyright holder. To view a copy of this licence, visit http://creativecommons.org/licenses/by/4.0/. 


\section{References}

1. Akman A, Sahillioğlu Y, Sezgin TM (2020) Generation of 3D human models and animations using simple sketches. Graphics Interface 2020 Conference, https://openreview.net/forum?id=ozFu9KivuQ

2. Arqub OA (2017) Adaptation of reproducing kernel algorithm for solving fuzzy Fredholm-Volterra integrodifferential equations. Neural Comput Applic 28:1591-1610

3. Arqub OA, Abo-Hammour Z (2014) Numerical solution of systems of second-order boundary value problems using continuous genetic algorithm. Inf Sci 279:396-415

4. Arqub OA, Al-Smadi M, Momani S, Haya T (2017) Application of reproducing kernel algorithm for solving second-order, two-point fuzzy boundary value problems. Soft Comput 21:7191-7206

5. Bermano A, Vaxman A, Gotsman C (2011) Online reconstruction of 3D objects from arbitrary crosssections. ACM Trans Graph 30(5):1-14 (Art ID. 113)

6. Botsch M, Kobbelt L (2004) An intuitive framework for real-time freeform modeling. ACM Trans Graph 23(3):630-634

7. Botsch B, Sorkine O (2008) On linear variational surface deformation methods. IEEE Trans Vis Comput Graph 14(1):213-230

8. Bouaziz S, Martin S, Liu T et al (2014) Projective dynamics: fusing constraints for fast simulation. ACM Trans Graph 33(4):1-11 (Art ID. 154)

9. Ding C, Liu L (2016) A survey of sketch based modeling systems. Front Comput Sci 10(6):985-999

10. Eitz M, Richer R, Boubekeur T et al (2012) Sketch-based shape retrieval. ACM Trans Graph 31(4):1-10 (Art. ID 31)

11. Funkhouser T, Kazhdan M, Shilane P et al (2004) Modeling by example. ACM Trans Graph 23(3):652-663

12. Gingold Y, Igarashi T, Zorin D (2009) Structured annotations for 2D-to-3D modeling. ACM Trans Graph 28(5):1-9 (Art. ID 148)

13. Gryaditskaya Y, Sypesteyn M, Hoftijzer JW, Pont S, Durand F, Bousseau A (2019) OpenSketch: a richlyannotated dataset of product design sketches. ACM Trans Graph 38(6):1-16 (Art ID. 232)

14. Guo X, Lin J, Xu K, Chaudhuri S, Jin X (2016) CustomCut: on-demand extraction of customized 3D parts with 2D sketches. Comput Graph Forum 35(5):89-100

15. Hahm D, Wojtan C (2016) Fast approximations for boundary element based brittle fracture simulation. ACM Trans Graph 35(4):1-11 (Art ID. 104)

16. Han X, Gao C, Yu Y (2017) DeepSketch2Face: A deep learning based sketching system for 3D face and caricature modeling. ACM Trans Graph 36(4):1-12 (Art ID. 126)

17. Huang H, Kalogerakis E, Yumer E, Mech R (2017) Shape synthesis from sketches via procedural models and convolutional networks. IEEE Trans Vis Comput Graph 23(8):2003-2013

18. Igarashi T, Matsuoka S, Tanaka H (1999) Teddy: a sketching interface for 3D freeform design. Proc ACM SIGGRAPH pp. 409-416

19. Johnston H, Leake C, Mortari D (2020) Least-squares solutions of eighth-order boundary value problems using the theory of functional connections. Mathematics 8:1-17. https://doi.org/10.3390/math8030397

20. Jones B, Thuerey N, Shinar T et al (2016) Example-based plastic deformation of rigid bodies. ACM Trans Graph 35(4):1-11 (Art ID. 34)

21. Kazmi IK, You LH, Yang XS et al (2015) Efficient sketch-based creation of detailed character models through data-driven mesh deformations. Comput Animat Virtual Worlds 26(3-4):469-481

22. Kraevoy V, Sheffer A, van de Panne M (2009) Modeling from contour drawings. In Proceedings of the 6th Eurographics Symposium on Sketch-Based Interfaces and Modeling (SBIM’09), pp. 37-44

23. Li B, Lu Y, Godil A, Schreck T, Bustos B, Ferreira A, Furuya T, Fonseca MJ, Johan H, Matsuda T, Ohbuchi R, Pascoal PB, Saavedra JM (2014) A comparison of methods for sketch-based 3D shape retrieval. Comput Vis Image Underst 119:57-80

24. Li C, Pan H, Liu Y, Tong X, Sheffer A, Wang W (2018) Robust flow-guided neural prediction for sketchbased freeform surface modeling. ACM Trans Graph 37(6):1-12 (Art ID. 238)

25. Li Y, Lei H, Lin S, Luo G (2018) A new sketch-based 3D model retrieval method by using composite features. Multimed Tools Appl 77:2921-2944

26. Liu S, Hou F, Hao A, and Qin H (2019) RegionSketch: interactive and rapid creation of 3D models with rich details. Proceedings of 2019 Pacific Graphics Conference, pp 1-6

27. Liu J, Lian Z, Xiao J (2020) Sketch based modeling and editing via shape space exploration. Multimed Tools Appl. https://doi.org/10.1007/s11042-020-08677-0

28. Lun Z, Gadelha M, Kalogerakis E, Maji S, Wang R (2017) 3D shape reconstruction from sketches via multi-view convolutional networks. In 2017 International Conference on 3D Vision (3DV), pp. 67-77

29. Madsen D (2016) Engineering drawing and design. CENGAGE Delmar Learning

30. Mezger J, Thomaszewski B, Pabst S, Straßer W (2009) Interactive physically-based shape editing. Comput Aided Geom Des 26:680-694 
31. Nealen A, Müller M, Keiser R, Boxerman E, Carlson M (2006) Physically based deformable models in computer graphics. Comput Graph Forum 25(4):809-836

32. Nealen A, Igarashi T, Sorkine T et al (2007) FiberMesh: Designing freeform surfaces with $3 \mathrm{D}$ curves. ACM Trans Graph 26(3):1-9 (Art. ID 41)

33. Olsen L, Samavati F, Sousa M, Jorge J (2009) Sketch-based modeling: a survey. Comput Graph 33(1):85103

34. Rivers A, Durand F, Igarashi T (2010) 3D modeling with silhouettes. ACM Trans Graph 29(4):1-8 (Art. ID 109)

35. Smirnov D, Bessmeltsev M, Solomon J (2019) Deep sketch-based modeling of man-made shapes. arXiv: $1906.12337 \mathrm{v} 1$

36. Sorkine O, Cohen-Or D, Lipman Y, et al. (2004) Laplacian surface editing. In Proceedings of the 2014 Eurographics/ACM SIGGRAPH Symposium on Geometry Processing, pp. 175-184

37. Wang B, Wu L, Yin K et al (2015) Deformation capture and modeling of soft objects. ACM Trans Graph 34(4):1-12 (Art ID. 94)

38. Xu K, Chen K, Fu H et al (2013) Sketch2Scene: sketch-based co-retrieval and co-placement of 3D models. ACM Trans Graph 32(4):1-12 (Art ID. 123)

39. Xu B, Chang W, Sheffer A, Bousseau A, McCare J, Singh K (2014) True2Form: 3D curve networks from 2D sketches via selective regularization. ACM Trans Graph 33(4):1-13 (Art ID. 131)

40. You LH, Zhang JJ, Comninos P (2004) Blending surface generation using a fast and accurate analytical solution of a fourth-order PDE with three shape control parameters. Vis Comput 20(2):109-214

41. You LH, Comninos P, Zhang JJ (2004) PDE blending surfaces with $C^{2}$ continuity. Comput Graph 28(6): 895-906

42. You LH, Chang J, Yang XS, Zhang JJ (2011) Solid modelling based on sixth order partial differential equations. Comput Aided Des 43:720-729

43. Zhang JJ, You LH (2002) PDE based surface representation - vase design. Comput Graph 26(1):89-98

44. Zhou W, Jia J (2019) A learning framework for shape retrieval based on multilayer perceptrons. Pattern Recogn Lett 117(1):119-130

45. Zhou W, Jia J, Jiang W, Huang C (2020) Sketch augmentation-driven shape retrieval learning framework based on convolutional neural networks. IEEE Trans Vis Comput Graph 24(3):1-14

46. Zimmermann J, Nealen A, Alexa M (2007) SilSketch: automated sketch-based editing of surface meshes. In Proceedings of the 4th Eurographics Workshop on Sketch-Based Interfaces and Modeling, pp. 23-30

47. Zou M, Holloway M, Carr N et al (2015) Topology-constrained surface reconstruction from cross-section. ACM Trans Graph 34(4):1-10 (Art ID. 128)

Publisher's note Springer Nature remains neutral with regard to jurisdictional claims in published maps and institutional affiliations.

\section{Affiliations}

\section{Lihua You ${ }^{1} \cdot$ Xiaosong Yang $^{1} \cdot$ Junjun Pan $^{2} \cdot$ Tong-Yee Lee $^{3} \cdot$ Shaojun Bian $^{1} \cdot$ Kun Qian ${ }^{4} \cdot$ Zulfiqar Habib $^{5}$ - Allah Bux Sargano ${ }^{5}$ - Ismail Kazmi ${ }^{6}$ • Jian J. Zhang ${ }^{1}$}

1 National Centre for Computer Animation, Bournemouth University, Fern Barrow, Wallisdown, Poole BH12 5BB, UK

2 State Key Lab of VR, Beihang University, 37 Xueyuan Road, Haidian District, Beijing 100083, People's Republic of China

3 National Cheng Kung University, East District, Tainan City, Taiwan 701

4 King's College London, Strand, London WC2R 2LS, UK

5 COMSATS University Islamabad, Lahore, Pakistan

6 Teesside University, Campus Heart, Southfield Rd, Middlesbrough TS1 3BX, UK 